\title{
ANTIREFLECTION COATING DESIGN FOR SERIES INTERCONNECTED MULTI-JUNCTION SOLAR CELLS
}

Author:

Daniel J. Aiken

Affiliation Sandia National Laboratories

Address MS 0752

Sandia National Laboratories

Albuquerque, NM, 87185

djaiken@sandia.gov

Tel. 505 284-6607

Fax 505 844-6541

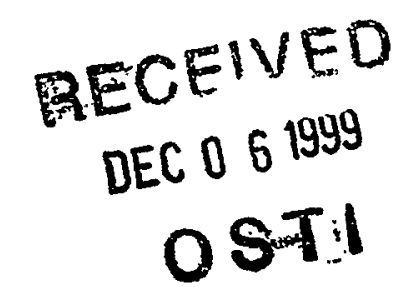

Sandia is a multi-program laboratory operated by Sandia Corporation, a - Lockheed Martin Company, for the U.S. Department of Energy under contract DE-AC04-94AL85000. 


\section{DISCLAIMER}

This report was prepared as an account of work sponsored by an agency of the United States Government. Neither the United States Government nor any agency thereof, nor any of their employees, make any warranty, express or implied, or assumes any legal liability or responsibility for the accuracy, completeness, or usefulness of any information, apparatus, product, or process disclosed, or represents that its use would not infringe privately owned rights. Reference herein to any specific commercial product, process, or service by trade name, trademark, manufacturer, or otherwise does not necessarily constitute or imply its endorsement, recommendation, or favoring by the United States Government or any agency thereof. The views and opinions of authors expressed herein do not necessarily state or reflect those of the United States Government or any agency thereof. 


\section{DISCLAIMER}

Portions of this document may be illegible in electronic image products. Images are produced from the best available original document. 


\section{SUMMARY}

AR coating design for multi-junction solar cells can be more challenging than in the single junction case. Reasons for this are discussed. Analytical expressions used to optimize AR coatings for single junction solar cells are extended for use in monolithic, series interconnnected multi-junction solar cell AR coating design. The result is an analytical expression which relates the solar cell performance (through $\mathrm{J}_{S c}$ ) directly to the AR coating design through the device reflectance. It is also illustrated how AR coating design can be used to provide an additional degree of freedom for current matching multi-junction devices.

\section{INTRODUCTION}

Bandgap engineering has been successfully used to develop high efficiency multi-gap 2and 3 -junction solar cells based on either III-V compounds $[1,2]$ or amorphous silicon alloys $[3,4]$ for space power and/or low cost flat plate applications. Multi-junction solar cells are also appealing candidates for use in concentrator systems due to their high efficiencies. As a result of this success there is considerable interest in furthering this concept with the development ultra-high efficiency 4 -junction solar cells $[5,6]$.

These multi-junction solar cells are primarily monolithically integrated, series interconnected devices, where the device Jsc is limited by the subcell with the lowest current generating capability. Current matching all subcells is therefore desirable and is accomplished by tailoring the subcell bandgaps and thicknesses to optimize light absorption.

Antireflection coating design for single junction solar cells has reached a high level of maturity and has been extensively reviewed in the literature. Proper antireflection (AR) coating design for multi-junction solar cells, however, is more challenging than in the single-junction case for several reasons. First, multi-junctions may convert a larger bandwidth of the solar spectrum to electrical energy than do single junction solar cells. This requires a more broadband AR coating. There are several exceptions to this rule, one of which is evident when comparing the larger 
spectral bandwidth of single junction silicon solar cells to $\ln \mathrm{GaP}_{2} / \mathrm{GaAs}$ multi-junction solar cells. In general, howeverr, the bandwidth of a solar cell is likely to increase as more junctions are added. Secondly, series interconnection of multiple subcells places additional demands on AR coating performance. In the single junction case the goal is to couple as much light into the device as possible. In the multi-junction case the additional requirement is to distribute that light to each subcell such that current matching is either maintained or improved. Thirdly, series interconnected multi-junctions are also likely to be more sensitive to variations in AR coating design because any variation in AR coating design away from the optimum will result in increased current mismatch.

These design challenges are illustrated with Figure 1 and Table I. In Figure 1 a typical single junction silicon solar cell (a) is compared to a hypothetical 4-junction solar cell (b) with subcell bandgaps chosen such that the device is current matched in the event of no reflection loss, i.e. a perfect AR coating. The internal quantum efficiency of each cell or subcell is multiplied by the AMO spectrum and represents the portion of the spectrum that each subcell converts to current in short circuit. The area under each curve is the integrated short circuit current (Jsc) in the event of zero reflectance $R(\lambda)$, as computed using the equation

$$
J_{s c}=q \int_{\lambda} F(\lambda) \cdot \operatorname{EQE}(\lambda) d \lambda=q \int_{\lambda} F(\lambda) \cdot \operatorname{IQE}(\lambda) \cdot[1-R(\lambda)] d \lambda
$$

where $F(\lambda)$ is the photon flux, $\operatorname{EQE}(\lambda)$ and $\operatorname{LQE}(\lambda)$ are the external and internal quantum efficiencies, respectively, and $R(\lambda)$ is the total device reflectance.

Also shown are the modeled reflectances of each device when coated with an optimized double layer antireflection (DLAR) coating that was designed using the techniques that will be described in this paper. All AR coating structures modeled in this paper consisted of unencapsulated $\mathrm{TiO}_{2} / \mathrm{Al}_{2} \mathrm{O}_{3}$. The interference fringing that is evident in the reflectance of the multi-junction device is a result of internal reflection at the numerous material interfaces.

Table I lists the resulting integrated Jsc values computed using Equation 1 and using three different modeled reflectances: $R(\lambda)=0, R(\lambda)$ of the optimized DLAR, and $R(\lambda)$ of the optimized 
DLAR red-shifted by $20 \mathrm{~nm}$. The integrated Jsc in the case $R(\lambda)=0$ can be regarded as the maximum achievable Jsc. In the-4-junction case the overall device Jsc is equal to the minimum subcell Jsc as a result of the implied series interconnection.

Table I Integrated short circuit currents for the two solar cells of Figure 1 as computed using Equation 1 with three different modeled reflectances.

\begin{tabular}{|c|c|c|c|c|c|c|c|c|}
\cline { 2 - 9 } \multicolumn{1}{c|}{} & \multicolumn{1}{|c|}{ Single junction (Fig. 1a) } & \multicolumn{5}{|c|}{ 4-junction (Fig. 1b) } \\
\cline { 2 - 9 } & $\begin{array}{c}\text { device } \\
\text { Jsc }\end{array}$ & $\begin{array}{c}\text { change in } \\
\text { device Jsc (\%) }\end{array}$ & $\begin{array}{c}\text { top cell } \\
\text { Jsc }\end{array}$ & $\begin{array}{c}2^{\text {nd }} \\
\text { cell }\end{array}$ & $\begin{array}{c}3^{\text {rd }} \\
\text { cell }\end{array}$ & $\begin{array}{c}\text { 4th } \\
\text { cell }\end{array}$ & $\begin{array}{c}\text { device } \\
\text { Jsc }\end{array}$ & $\begin{array}{c}\text { change in } \\
\text { device Jsc (\%) }\end{array}$ \\
\hline$(R(\lambda)=0),\left(\mathrm{mA} / \mathrm{cm}^{2}\right)$ & 44.83 & & 18.12 & 18.09 & 18.09 & 18.07 & 18.07 & \\
\hline optimized DLAR $\left(\mathrm{mA} / \mathrm{cm}^{2}\right)$ & 42.50 & -5.2 & 16.51 & 16.56 & 16.60 & 16.48 & 16.48 & -8.8 \\
\hline red shifted refl. $\left({\left.\mathrm{mA} / \mathrm{cm}^{2}\right)}^{2}\right)$ & 42.48 & -0.05 & 16.16 & 16.74 & 16.53 & 16.62 & 16.16 & -1.9 \\
\hline
\end{tabular}

As evident from Table I, the 4-junction optimized for a current limiting third subcell.device loses $8.8 \%$ of its maximum achievable Jsc as a result of reflection loss from an optimized DLAR coating, as opposed to $5.2 \%$ loss for the single junction device. For the same general DLAR coating structure, the 4-junction device loses a larger percentage of the available current due to reflection than the single junction device. This occurs for two reasons: The limited bandwidth of a DLAR coating is adequate for the silicon cell but is inadequate for the 4-junction cell, which responds out to the band edge of the germanium bottom cell. This is evident from the relatively high reflectivity at both ends of the 4-junction spectral range and the significant reflectance near the middle of the spectral range. Secondly, because subcell 4 limits the current of every other subcell, all other subcells have effectively suffered the same reflectance loss as subcell 4 . The AR coating is therefore only as good as the performance of the worst subcell.

The effect of varying the AR coating thicknesses away from their optimized values can be simulated by shifting the modeled reflectance to longer or shorter wavelengths. The reflectances of the optimized AR coatings for both devices have been red-shifted by $20 \mathrm{~nm}$ to simulate AR coating variations, and the resulting integrated currents are shown in the last row of Table I. The single junction, device loses almost no additional current due to this red shift, while the Jsc of the 
4-junction device drops nearly $2 \%$ with respect to the optimized device. This is because the AR coating must be equally effective across all subcells. Shifting the reflectance away from the optimum is more likely to result in significant reflectance loss because each subcell in the multijunction device responds to a smaller wavelength range than the single junction device.

Although not all multi-junction solar cells will show losses of the same magnitude as those of the 4-junction current matched device of Figure 1, multi-junction solar cells will in general require higher performance AR coatings and will be more sensitive to variations in the AR coating design. A formal procedure for designing AR coatings for these multi-junctions is therefore necessary. The scope of this paper is not to establish which AR coating designs are appropriate for any given multi-junction or to compare the performance of specific designs, but rather to describe an effective design approach for optimizing AR coatings and evaluating their effectiveness. This can be accomplished by extending the analytical expressions often used in single junction AR coating design to be applicable to multi-junction design. Antireflection coating design can then be used in conjunction with subcell thickness adjustments to provide a greater flexibility for achieving current matching.

\section{SINGLE JUNCTION AR COATING DESIGN}

The goal in developing high performance antireflection (AR) coatings for solar cells is to maximize the light generated current. Equation 1 is typically used to calculate an integrated current in terms of the measurable $\operatorname{EQE}(\lambda)$. This equation can also be used to design an optimum AR coating because it directly relates $J_{S C}$ to the AR coating design through the parameter $R(\lambda)$.

Another parameter used in AR coating design and optimization is the solar weighted reflectance (SWR) as first introduced by Redfield [7]. The SWR is defined as

$$
S W R=\frac{\int F(\lambda) \cdot \operatorname{IQE}(\lambda) \cdot R(\lambda) \cdot d \lambda}{\int F(\lambda) \cdot \operatorname{IQE}(\lambda) \cdot d \lambda}=\frac{\text { useable photons reflected }}{\text { total useable photons }}
$$


The term useable photons refers to photons that, if not reflected, will contribute to the photocurrent as a result of the collection of an electron. Minimizing the SWR will minimize the number of useable photons that are reflected. Equation 1 can be manipulated and inserted into Equation 2, resulting in an alternative expression for the SWR given by

$$
s W R=1-\left(\frac{J_{s c}}{\left.J_{s c}\right|_{R(\lambda)=0}}\right),
$$

where $\left.J s c\right|_{R(\lambda)=0}$ is the available short circuit current, i.e. the short circuit current in the event of no reflection loss. Minimizing the SWR using Equation 2 is equivalent to maximizing the short circuit current using Equation 1, as suggested by Equation 3. The SWR is a convenient parameter for evaluating the effectiveness of an AR coating because it is equal to the fraction of the available short circuit current that is lost due to reflection. These equations assume that the $\operatorname{IQE}(\lambda)$ is independent of the device reflectance $R(\lambda)$ and that negligible parasitic absorption occurs in the AR coating structure.

Equations 1 or 2 can be employed in AR coating design by using optical theory [8] to calculate the device reflectance as a function of the layer thicknesses in a general AR coating structure. In this case the term $R(\lambda)$ is a modeled reflectance and requires that the optical properties of all materials in the device structure are accurately known such that the modeled reflectance closely matches the actual reflectance. Numerous examples of the application of Equations 1 or 2 are found in the literature [9-11].

\section{MULTI-JUNCTION AR COATING DESIGN}

Maximizing the light generated current in series interconnected multi-junction solar cells places an additional design requirement on AR coatings. In this case the goal is not only to couple the maximum amount of light into the device, but also to distribute that light to each subcell such 
that the device is as closely current matched as possible. Stated alternatively, the goal is to maximize the light generated current of the current-limiting subcell.

Equation 1 could also be used to design AR coatings for series connected multi-junction solar cells, provided that the term IQE $(\lambda)$ is replaced with $\operatorname{IQE}_{x}(\lambda)$. Here $\operatorname{IQE} E_{x}(\lambda)$ is the internal quantum efficiency of the current limiting subcell as determined from spectral response measurements carried out using the spectrally selective light biasing technique described by Burdick and Glatfelter [12]. The problem in determining the correct IQE $(\lambda)$ is that, in searching for an AR coating design that maximizes Jsc, $R(\lambda)$ will change and may in turn change which subcell limits the current, especially if the subcells are already closely current matched in the ideal case of $R(\lambda)=0$. To eliminate this difficulty, Equation 1 can be modified to accommodate multi-junction AR coating design such that the correct current limiting subcell for any given AR coating design does not have to be explicitly known. A more general form of Equation 1 which is also applicable to - multi-junction AR coating design is then given by

$$
J_{s c}=\operatorname{MIN}\left[J_{\mathbf{S c}_{1}}, J_{\text {sc } 2}, \text { etc. }\right]=\operatorname{MaN}\left[q \int_{\lambda} F(\lambda) \cdot \operatorname{IQE} E_{1}(\lambda) \cdot[1-R(\lambda)] d \lambda, q \int_{\lambda} F(\lambda) \cdot \operatorname{IQ} E_{2}(\lambda) \cdot[1-R(\lambda)] d \lambda \text {, etc. }\right]
$$

(4)

where $J_{S C 1}$ and $J_{S C 2}$ are the short circuit currents that subcells 1 and 2 are capable of generating, respectively, if not current limited by some other subcell. A solar weighted reflectance for the multi-junction case could be defined by inserting Equation 4 into Equation 3, and would again equal the fraction of available short circuit current that is lost to reflection.

The most difficult aspect of using Equation 4 in practice is calculation of the modeled reflectance. Compiling the optical properties of all relevant materials is a difficult task due to the many different materials and layers used, especially in multi-junctions made of III-V compounds. All modeling performed in this analysis used the best available optical constants as a function of wavelength for all relevant semiconductor or dielectric layers. Additionally, because multi-junction cell structures are optically very complicated, analytic expressions for $R(\lambda)$ quickly become unmanageable and computer simulation is required. For example, all AR coating optimization 
reported here was done using FILMSTAR DESIGN optical thin film software from FTG Associates [13].

\section{AR COATING DESIGN AS A CURRENT MATCHING TECHNIQUE}

Multi-junction subcells that are already well current matched before AR coating deposition require a reflectance that is as low and flat as possible across the spectral range of interest such that current matching is maintained. The primary technique for current matching a multi-junction solar cell is by adjusting the individual subcell thicknesses. This technique is not infinitely flexible because the absorption in any subcell is dependent not only on its own thickness but also on the thickness of all subcells above it. Furthermore, increasing a subcells thickness to values significantly greater than the base diffusion length will not enhance that subcell's current generating capability. This imposes an upper limit on the useful thickness of any subcell in terms of current matching.

Proper AR coating design can provide an additional degree of freedom for current matching multi-junction devices by minimizing the reflectance in spectral regions where the corresponding subcells are current limiting the device, and trading that for higher reflectivity where other subcells have current to spare. Equation 4 will automatically perform this task by optimizing the AR coating design so as to maximize the current generating capability of the current limiting subcell.

This technique is illustrated with Figures 2 and 3. Shown in Figure 2 are the individual IQE curves of a typical 2-junction InGaP/GaAs multi-junction that is nearly current matched. Also shown is the modeled reflectance of a simplified cell structure with double layer AR coating that was optimized using Equation 4. It is apparent that the AR coating has been properly optimized

such that reflectance loss is divided equally between both subcells, thereby keeping the multijunction current matched.

In Figure 3 a current mismatch scenario has been modeled by lowering the IQE of the top subcell. The AR coating optimized for this case has traded higher reflectance in the bottom cell 
spectral region for lower reflectance in the top cell region, thereby assisting in current matching this multi-junction. Table II lists the simulated short circuit current densities for the devices in Figures 2 and 3 . The optimized AR coating for the current matched subcells of Figure 2 has resulted in both subcells possessing equal current loss due to reflection. This results in a maximum Jsc for the multi-junction. The optimized AR coating for the top subcell limited multijunction of Figure 3 has traded higher reflectivity for the bottom subcell in exchange for low reflectivity for the current limiting top subcell. This results in a lower current lost due to reflection for the top subcell $\left(0.48 \mathrm{~mA} / \mathrm{cm}^{2}\right)$ and maximizes the multi-junction Jsc.

Table II Short circuit current densities for the 2-junction solar cells simulated in Figures 2 and 3.

\begin{tabular}{|c|c|c|c|c|c|}
\cline { 2 - 3 } \multicolumn{1}{c|}{} & \multicolumn{2}{c|}{ Current matched (Figure 2) } & & \multicolumn{2}{c|}{ Current mismatched (Figure 3) } \\
\cline { 2 - 3 } \cline { 5 - 5 } \multicolumn{1}{c|}{ Jsc $(R(\lambda)=0),\left(m A / c^{2}\right)$} & 17.95 & 18.05 & & 15.68 & 18.05 \\
\hline Jsc, optimised DLAR, $\left(\mathrm{mAv} \mathrm{cm}^{2}\right)$ & 17.22 & 17.22 & & 15.20 & 16.39 \\
\hline Jsc lost to reflectance, $\left(\mathrm{mA} / \mathrm{cm}^{2}\right)$ & 0.73 & 0.83 & & 0.48 & 1.66 \\
\hline
\end{tabular}

Figure 4 illustrates the current matching technique for the hypothetical 4-junction solar cell of Figure 1b. Here the $\operatorname{IQE}(\lambda)$ of subcell 3 has been lowered to simulate a current mismatch situation. In the limiting case of no reflectance loss this multi-junction is current limited by subcell 3, as suggested by Table III. This multi-junction is likely to be more difficult to current match by adjusting subcell thicknesses due to the larger number of subcells and the limited flexibility afforded by changing subcell thicknesses as discussed previously. The AR coating which optimizes this multi-junction performance is also shown in Figure 4. Current matching has been assisted by minimizing the current that subcell 3 has lost to reflection as shown in Table III. 
Table III Short circuit current densities for the 4-junction solar cell simulated in Figure 4.

\begin{tabular}{|c|c|c|c|c|}
\cline { 2 - 5 } \multicolumn{1}{c|}{} & Top cell & 2nd cell & 3rd cell & Bottom cell \\
\hline Jsc $(R(\lambda)=0),\left(\mathrm{mA} / \mathrm{cm}^{2}\right)$ & 18.12 & 18.09 & 17.29 & 18.07 \\
\hline Jsc, optimized DLAR, $\left(\mathrm{mA} / \mathrm{cm}^{2}\right)$ & 16.61 & 16.72 & 16.44 & 16.32 \\
\hline Jsc lost to reflectance, $\left(\mathrm{mA} / \mathrm{cm}^{2}\right)$ & 1.51 & 1.37 & 0.85 & 1.75 \\
\hline
\end{tabular}

\section{CONCLUSIONS}

Series interconnected multi-junction solar cells will require AR coatings with higher performance than those currently used for single junction solar cells. In the single junction case the goal is to couple the maximum amount of light into the cell. For series connected multijunction devices current matching is also a critical design goal. The goal in designing antireflection (AR) coatings for series interconnected multi-junction solar cells is therefore not only to couple the maximum amount of light into the device, but also to distribute that light to each subcell such that the device is as closely current matched as possible. Analytical expressions used to optimize AR coatings for single junction solar cells have been extended for use in monolithic, series interconnnected multi-junction solar cell AR coating design. The result is an analytical expression which couples the solar cell performance (through $\mathrm{J}_{\mathrm{SC}}$ ) directly to the AR coating design through the device reflectance. This expression assumes that the reflectance of a multi-junction device structure can be accurately modeled and therefore requires kniowledge of optical constants for all relevant materials in the multi-junction. Proper AR coating design can also be used to provide an additional degree of freedom for current matching multi-junction devices.

\section{REFERENCES}


[1] D. Keener, D. Marvin, D. Brinker, H. Curtis, P. Price, "Progress Toward Technology Transition of GalnP $\mathrm{P}_{2} / \mathrm{GaAs} / \mathrm{Ge}$ Multijunction Solar Cells", Proceedings $26^{\text {th }}$ IEEE PVSC, 787792, (1997).

[2] B. Cavicchi, J. Ermer, D. Krut, D. Joslin, M. Gillanders, D. Zemmrich, "250,000 Watts of GalnP2/GaAs/Ge Dual Junction Production", $2^{\text {nd }}$ World Conference on PV Energy Conversion, 3515-3519, (1998).

[3] J. Yang, A. Banerjee, T. Glatfelter, S. Sugiyama, S. Guha, "Recent Progress in Amorphous Silicon Alloy leading to $13 \%$ Stable Cell Efficiency", Proceedings $26^{\text {h }}$ IEEE PVSC, 563-568, (1997).

- [4] S. Guha, J. Yang, A. Banerjee, T. Glatfelter, G. Vendura, A. Garcia, M. Kruer, “Amorphous Silicon Alloy Solar Cells for Space Applications", $2^{\text {nd }}$ World Conference on PV Energy Conversion, 3609-3613, (1998).

[5] H. Hou, K. Reinhardt, S. Kurtz, J. Gee, A. Allerman, B. Hammons, P. Chang, E. Jones, "Novel InGaAsN pn Junction for High-Efficiency Multiple-Junction Solar Cells", $2^{\text {nd }}$ World Conference on PV Energy Conversion, 3600-3603, (1998).

[6] D. Friedman, J. Geisz, S. Kurtz, J. Olson, "1-eV GalnNAs Solar Cells for Ultra High Efficiency Multijunction Devices", $2^{\text {nd }}$ World Conference on PV Energy Conversion, 3-7, (1998).

[7] D. Redfield, "Method for Evaluation of Antireflection Coatings", Solar Cells, vol. 3, 27-33, (1981).

[8] H.A. MacLeod, Thin Film Optical Filters, McGraw-Hill, 1989. 
[9] J. Zhao and M.A. Green, "Optimized Antireflection Coatings for High Efficiency Silicon Solar Cells", IEEE Transactions on Electron Devices, vol. 38, no. 8, 1925-1934, (1991).

[10] P. Doshi, G.E. Jellison, and A. Rohatgi, "Characterization and Optimization of Absorbing Plasma-Enhanced Chemical Vapor Deposition Deposited Antireflection Coatings for Silicon Photovoltaics", Applied Optics, vol 36, no. 30, 7826-7837, (1997).

[11] D. Bouhafs, A. Moussi, A. Chikouche, J.M. Ruiz, "Design and Simulation of Antireflection Coating Systems for Optoelectronic Devices: Application to Silicon Solar Cells", Solar Energy Materials and Solar Cells, 79-93, (1998).

- [12] J. Burdick and T. Glatfelter, "Spectral Response and I-V Measurements of Tandem Amorphous Silicon Alloy Solar Cells", Solar Cells, vol. 18, 301-314, (1986).

[13] FILMSTAR DESIGN version 2.0 from FTG Software Associates, P.O. Box 579, Princeton, N.J. 08542. 

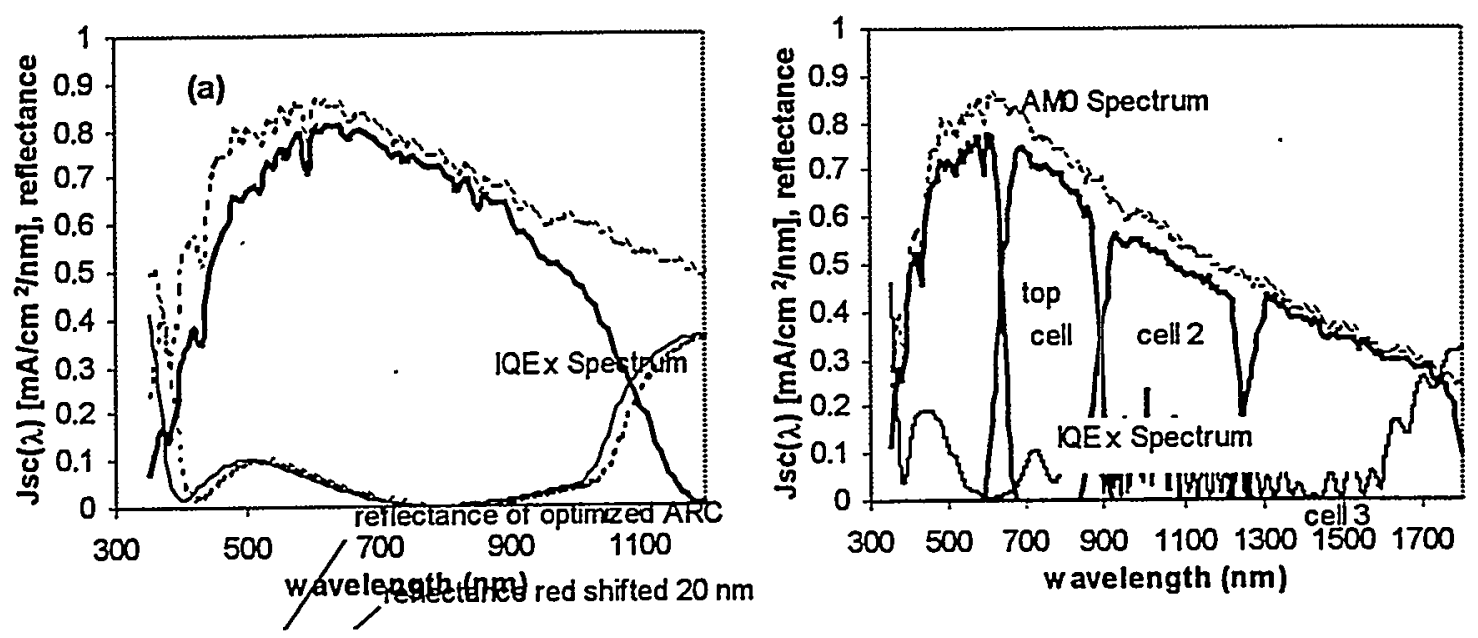


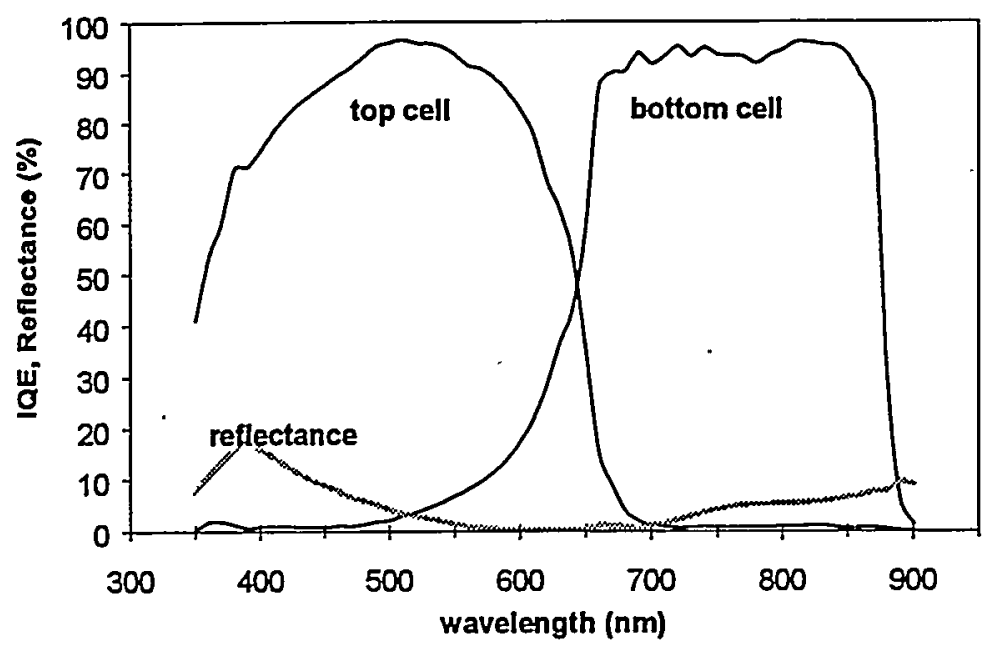




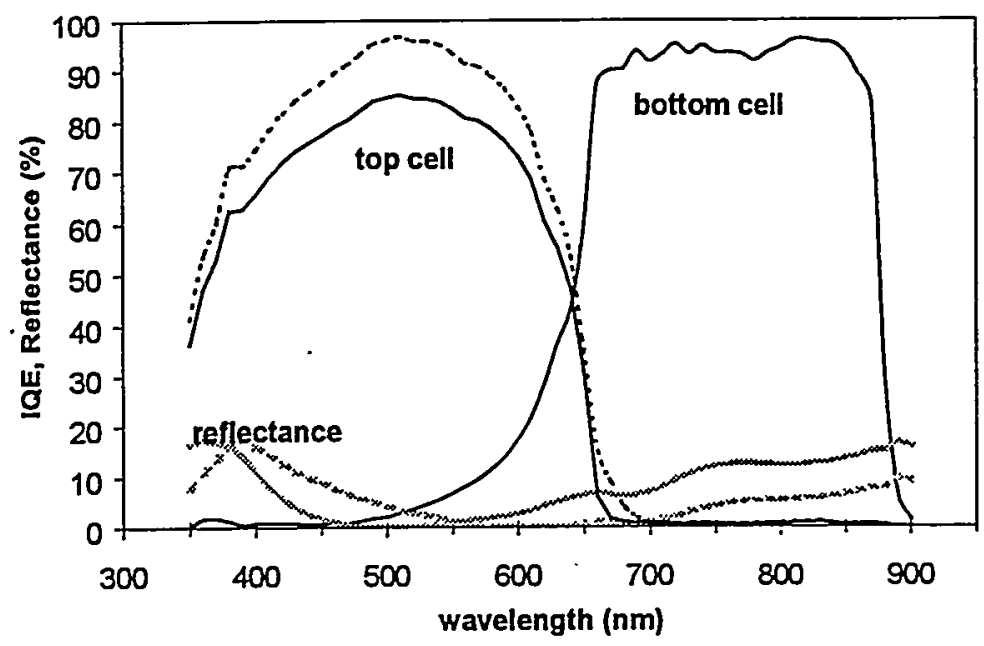




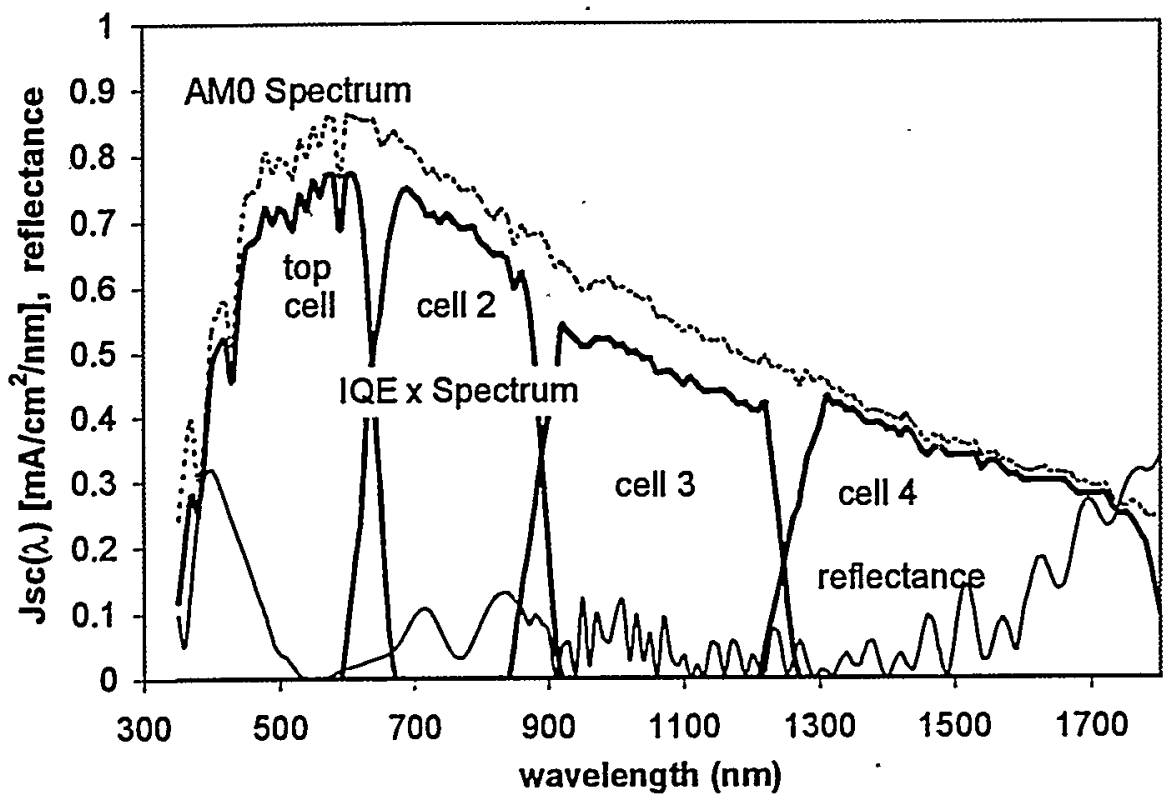


Figure 1 Graphic representation of data used to compute the integrated short circuit current of a single junction silicon solar cell (a) and a 4-junction solar cell (b) using Equation 1.

Figure 2 Reflectance and IQE of a 2-junction solar cell with an AR coating optimized for current matched subcells.

Figure 3 Reflectance and IQE of a 2-junction solar cell with an AR coating optimized for a current limiting top subcell. The top cell IQE and optimized reflectance from Figure 2 are shown as dashed lines for comparison.

Figure 4 Reflectance of a simulated future 4-junction solar cell with an AR coating optimized for a current limiting third subcell. 